Physical Sciences | Professor Michael D. Heagy

\title{
The power of light:
}

Production of solar fuels

Harnessing the power of
the sun as a source of energy
is highly appealing for many
reasons, but primarily because
it is a renewable, clean source
of energy. Plants are aready
incredibly proficient at not just
converting light to useable
energy but also at storing that
energy in the form of glucose,
which can be considered a
solar fuel. Professor Michael
D. Heagy at New Mexico Tech
has taken inspiration from plant
photosynthesis to develop
new materials, designed to
address some of the challenges
involved in efficiently converting
and storing solar energy.

\section{hen it comes to solar
energy conversion, plants
are the experts. Through} photosynthesis, plants can use light energy absorbed from the sun, in combination with carbon dioxide and water, to create glucose and oxygen. Despite photosynthesis being a complex, multi-step process, plants are able to efficiencies, owing to their highly adapted network of molecular machinery.

Glucose, the type of sugar produced during photosynthesis, is a very energymain purposes. Firstly, glucose provides the chemical building blocks for the plant

a plant's abilities for light harvesting, conversion and storage by breaking the photosynthetic process into two key steps. Water splitting, where the key goal is to oxidise water into hydrogen and oxygen, followed by the reduction fyrbon dioxide to allow solar fuel synthesis. Professor Michael D. Heagy team are experts at designing materits tailored to promote the conversion of carbon dioxide to solar fuels using the power of light alone.

\section{SOLAR FUELS}

Recreating the energy conversion efficiency of plants in the laboratory with artificial photosynthesis is a tricky task

Liquid solar fuels have an advantage not just in terms of their energy density but can also be used as chemical feedstocks. to be able to grow. Secondly, plants also exploit glucose as part of respiration, which inulo coplants and hinans, locked in the glucose fuel into a useable form.

Trying to mimic the incredible abilities of plants to convert and store the energy from sunlight has inspired the field of artificial photosynthesis. Artificial photosynthesis attempts to recreate

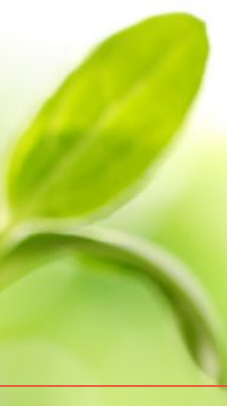

as well as overcoming one of the other issues with solar energy: how to provide continuous, uninterrupted energy supply from solar sources? The key to this energy, which is often done by using photoinduced chemical processes to create solar fuels.

Probably the most famous example of a solar fuel, and the one created during the water splitting stage of natural photosynthesis, is hydrogen. After using light-induced processes to form the hydrogen, this then acts as a fuel reservoir as the hydrogen can later be burnt or converted to release the stored energy when required. However, while hydrogen is now being used directly as a fuel by sydrogen por fued cars, is n not anideal hydrogen needs to be highly pess
Silver nanoparticles act as plasmonic sensitiser via $\mathrm{Ag} / \mathrm{Cu}_{2} \mathrm{O}$ nanocomposite and augment solar-driven bicarbonate formate conversion.
A

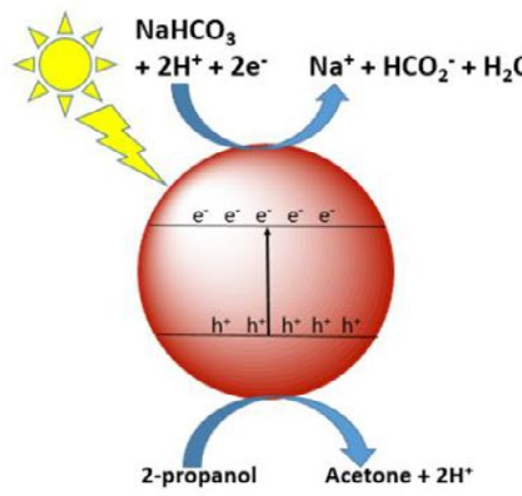

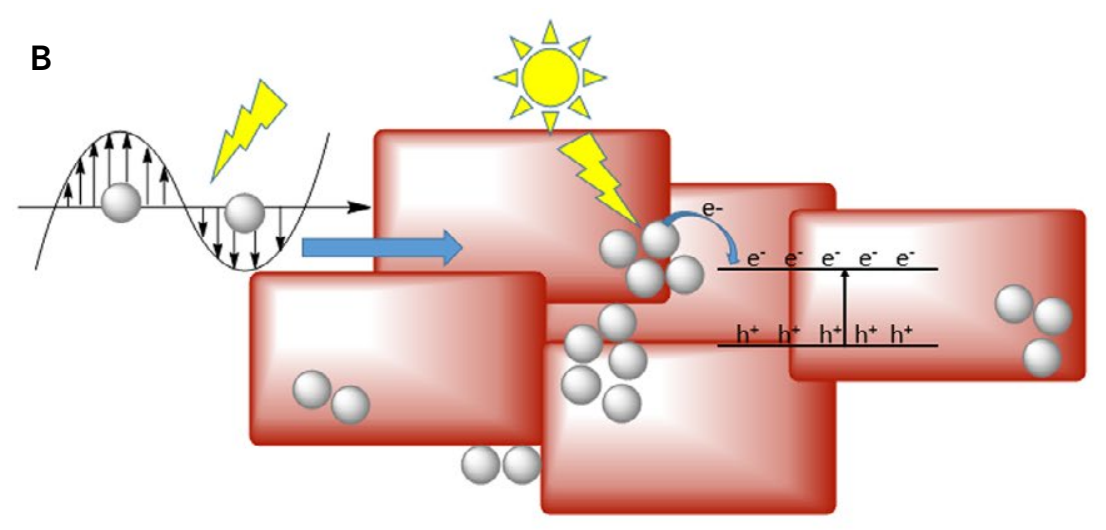

\section{Proposed mechanis
to semiconductor.)}

and is difficult to store safely owing to its flammability in using photochemical processes to create other kinds of solar fuels, including finding ways to efficiently produce formate, the negatively charged versio of the simplest carboxylic acid, formic acid. Producing formate and other liquid fuels not only is advantageous in terms of their superior energy density, but they can also be used as chemical feedstocks. Part of our global reliance on fossil fuels is not just using them as a source of energy, but also as chemical feedstocks for the manufacture of plastics and other chemicals. The idea of using methanol fossil fuels, for both a a synthesis, has been championed by Olh, 'menthe

MAKING MATERIALS

Professor Heagy's research focuses on designing photocatalysts and novel nanomaterials with specially designed structures for the reduction of bicarbonate to solar fuels. Bicarbonate exists in equilibrium with dissolved $\mathrm{CO}_{2}$ and is the predominant species at neutral $\mathrm{pH}$. While plants have specific cellular and chemical architectures adapted for all the stages of the photosynthetic cycle, in the laboratory, driving the same processes requires the use of photocatalysts.
This is why Professor Heagy is interested and dime thyl eher as alternatives for the chemical reactants on their surface. One photocatalyst that Professor Heagy Then, when they are illon their surface. photocatalysthelps to change charge distribution of electrons betwe itself and the reacting molecules. This process results in significant acceleration of the rate of chemical reactions such as the reduction of carbon dioxide.

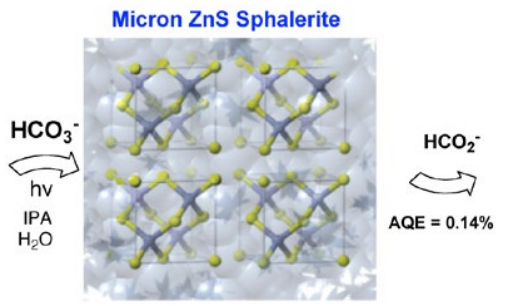

One photocatalyst that Professor Heagy has been investigating for solar fuel production is irn oxide. In his tean's found that the photocatalytic activity for reducing bicarbonate to formate is strongly dependent on the shape and structure of the material. By using
Two crystal forms of ZnS were synthesised and evaluated for their photochemical properties. The
wurtzite a rystal f form showed the highest AOE of $0.9 \%$ when using IPA as the whole scavenger. The AOE
increased

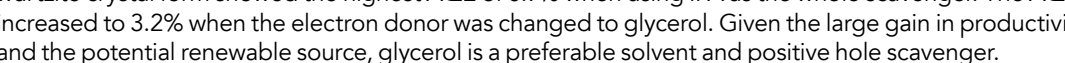

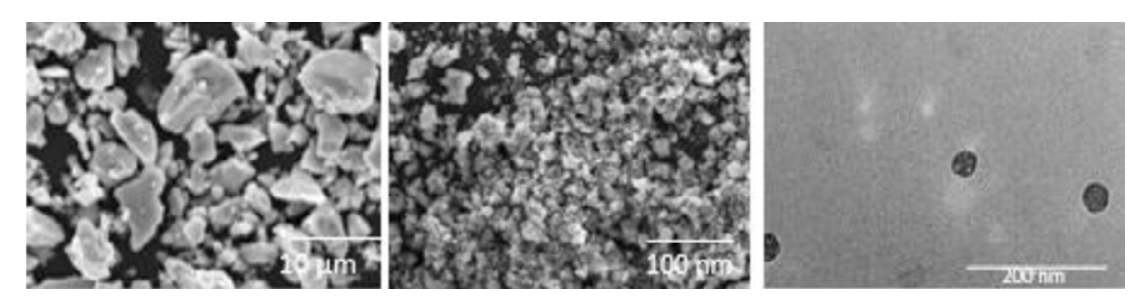

SEM image of (left) micron $\mathrm{Cu}_{2} \mathrm{O}$, and (contre) nano $\mathrm{Cu}_{2} \mathrm{O}$. (right) TEM images of nano $\mathrm{Cu}_{2} \mathrm{O}$ and $\mathrm{Ag} / \mathrm{Cu}_{2} \mathrm{O}$.

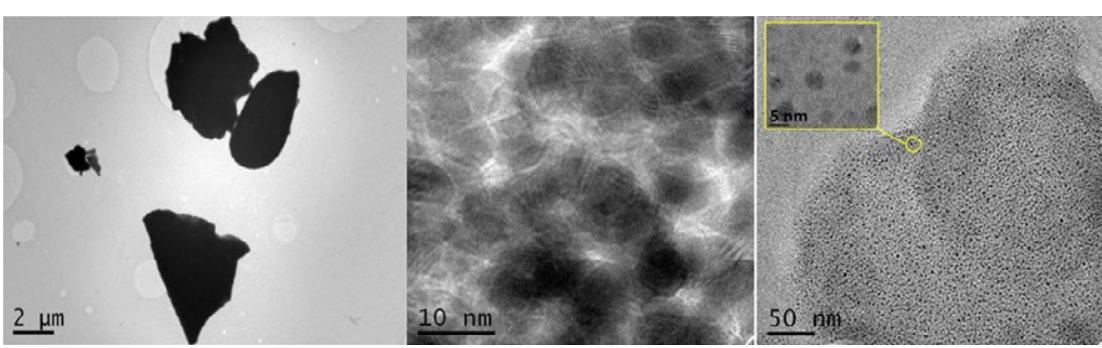

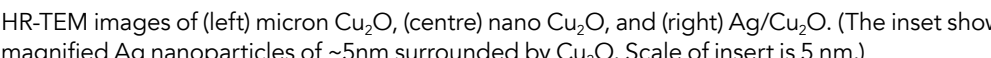



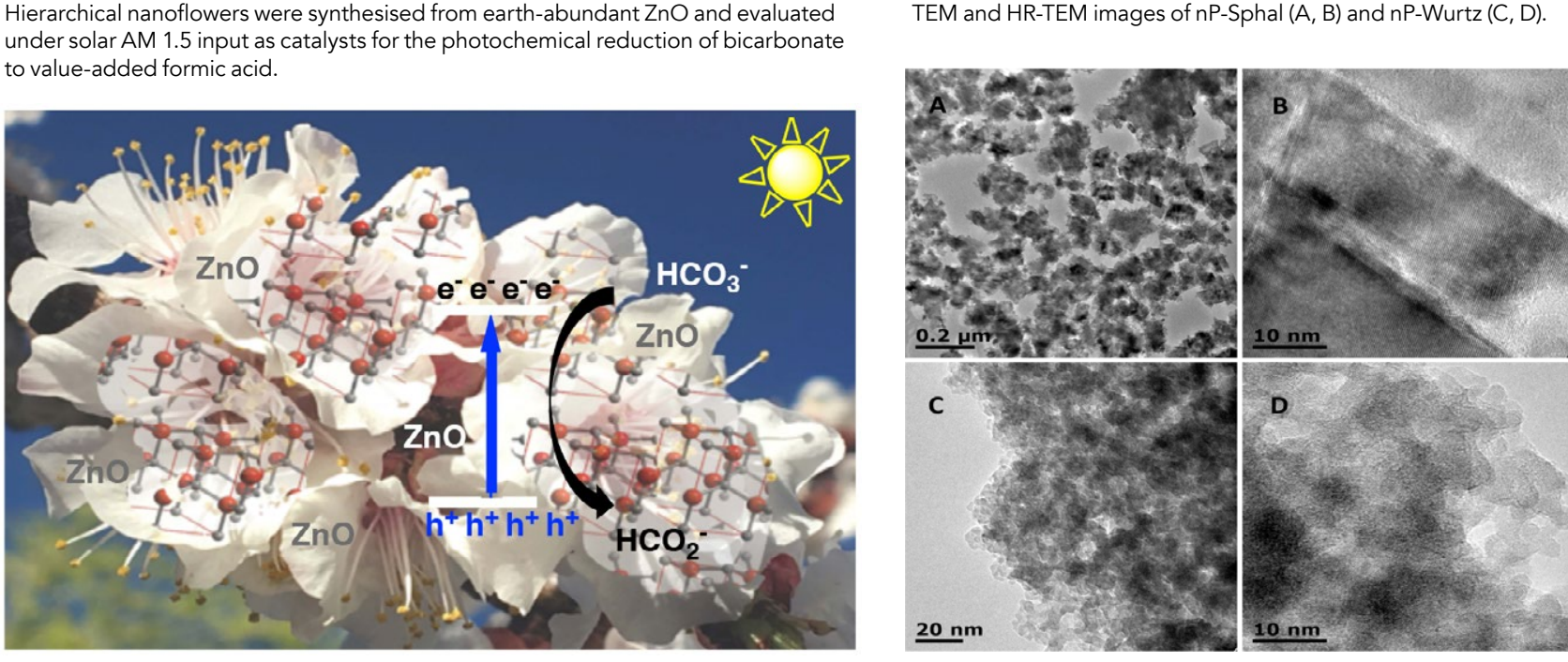

arrangements for the iron and oxygen atoms that gave the largest surface with, they could significanty inact whe productivity of the catalysts for the production of formate. Iron oxide is also an appealing choice of materia for this type of application as it is nontoxic and low-cost.

\section{THE NEW ECONOMY}

only material that looks like a promising candidate for photoreducing carbon dioxide to formic acid. Professor Heagy's group has also been investigating zinc sulfide structures and copper oxides to see whether thes would be more appealing photocatalysts for the production of formate and how the mechanism of the reaction differs, or is influenced by the shape of the particles, not just by changes in the has shown that exotic nanostructures, such as nanorods, nanobelts and nanoflowers, seem to be better

Investigating how and where the carbon dioxide molecules bind to the surface will help to drive intelligent design of photocatalysts.

catalysts than just having amorphous, This is important for such photocatalytic unstructured nanoparticles.

While Professor Heagy's group are developing new ways of controlling the miniature architectures of

nanoparticles, a lot of their research involves really trying to understand wo organic compounds using metal oxides photocatalysts work for these reduction was first reported in 1979, it has become the dissolve a wery hot area of research recently. With bind to the surbo woxide molecules more intelligent, whelp to dive nanoparticle structures. Alongside this, in they are also investigating whether changing the solvent environment for - used for such processes, we are one step

the photocatalysis also has an impact closer to realising the methanol economy. on the yield and rates of reaction.
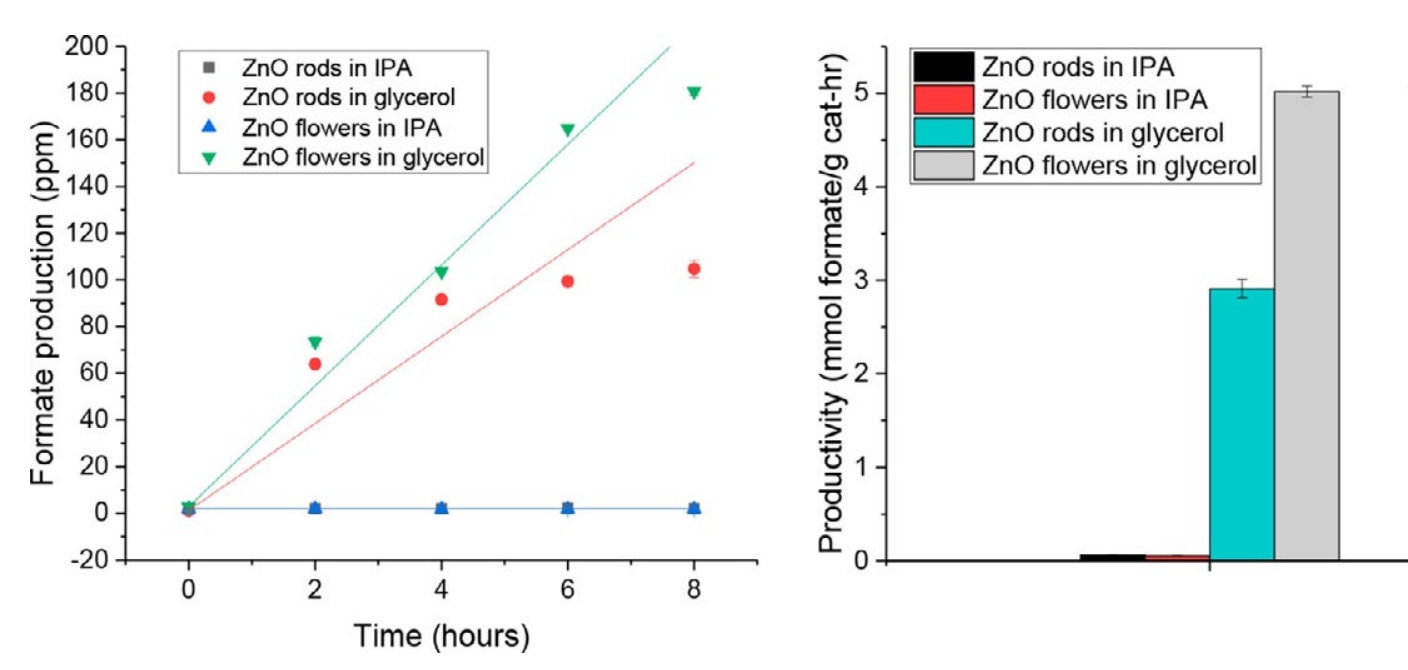

Formate production in ppm
with Zno rods and flowers in 2.-propanol and glycero
leetrland productivty (right

\section{Behind the Research} Professor Michael D. Heagy

E: Michael.heagy@nmt.edu T: +15758356185 W: https://sites.google.com/nmt.edu/heagyhome-page/home

Research Objectives

Professor Michael D. Heagy's work on novel nanostructures aims to improve the efficiency of solar fue

\section{Detail}

DrMichael Heagy

mentofChemistry

801 Leroy Place

Daniel H. Lopez Chemistry Rm 115

Socorro

USA

Bio

Michael D. Heagy received an AB degree in Chemistry from Franklin \& Marshall College, Lancaster, Pennsylvania and PhD under the direction of Nobel Laureate George A. Ola at the University of Southern California, Los Angeles. At the Massachusetts Institute of Technology, he conducted postdoctoral research with Prof Julius Rebek, Jr.

Funding

(n)

Collaborators

ging Pan

- Dr Sanchari Chowdhury

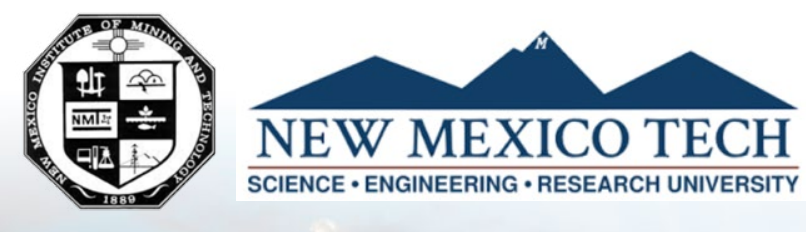

\section{References}

Leonard D., Pan H. and Heagy M., (2015), Photocatalyzed Reduction of Bicarbonate to Formate: Effect of ZnS Crystal Structure and Positive Hole Scavenger, ACS Applied Materials and Interfaces, 7, 24543-24549

Pan H., Chowdhury S., Premachandra D., Olguin S., Heagy M., (2018), Semiconductor Photocatalysis of Bicarbonate to Solar Finels. Chemistry and Engineering, 6, 1872-1880

Pan H., Martindale K., Heagy M., (2018), Iron Oxide Nanostructures for the Reduction of Bicaton Oxide Fuels, Topics in Catalysis, 61, 601-609

Pan H., Risley V., Martindale K., Heagy M.D., (2019) Perarchical Znc Oxide Nanostructures for the Photochemical Reduction of Bicarbonate to Solar Fuels, ACS Sustainable Chemistry and Engineering, 7, 1210-1219

\section{Personal Response}

\section{What are the remaining impediments to implementing} the meth

II Carbon dioxide capture remains a significant science con automobile exhaust, and other diluted sources are difficult to capture. Given the hydrophilic nature of methanol, if no such as aluminium and zinc. Existing pipelines designed for petroleum products cannot handle methanol Until new pipeline infrastructure can be built, or existing pipelines are retrofitted for methanol transport, methanol requires
shipment at higher energy cost in trucks and trains. 